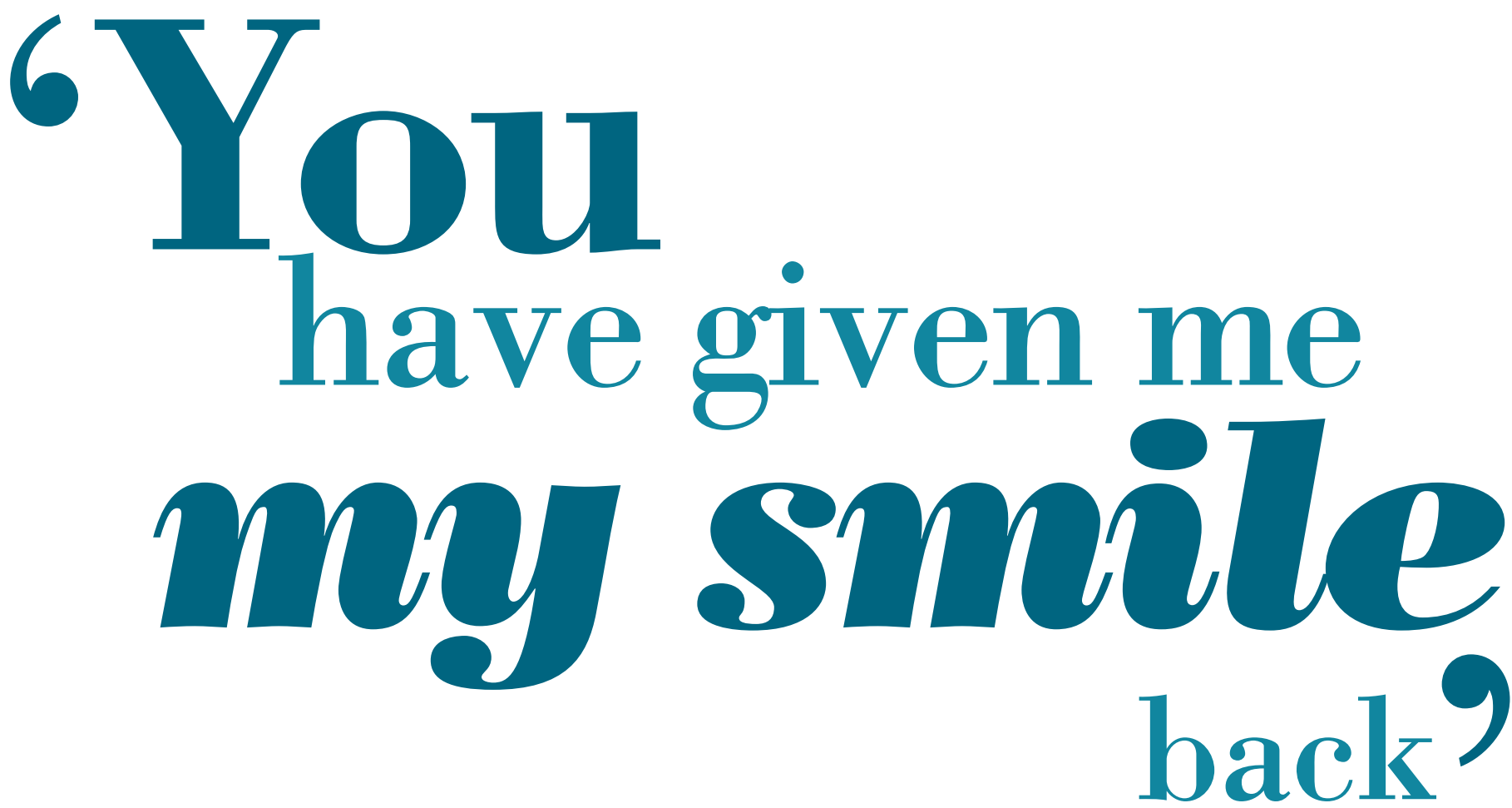

Dental nurse

Nicola George*

works alongside an

implantologist. Here

she tells us what her

job involves and

the impact implants

can have on a

patient's life.

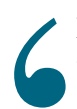

Denture?' 'Bridge?' 'Leave the gap?'

'Cosmetic?' 'Or functional?'

These are the questions that I ask patients when I discuss their options as new patients to the Dental Implant Clinic in Bath where I work.

The patients we see at the Dental Implant Clinic have usually tried most of the above scenarios in their dental history. The most common complaint from patients is that their denture is unbearable. They dislike eating and talking with them and having to remove them to clean. Patients who have lost a single tooth in an accident or for periodontal problems also come into the practice with low self esteem. Most people at this point question how patients coped with partial and full dentures before implants were available. The answer is that patients no longer have to struggle with dentures that cover the palate of their mouth, as there is another option.

\section{Changing lives}

Most of the patients we see feel very guilty coming into the practice to discuss having what might be considered cosmetic treatment. At this point the patient is very vulnerable and disheartened at the thought of having further treatment carried out in their mouth.

This is where the dental implant nurse's role begins. The best thing about being a dental nurse for an implantologist is that every day you look forward to changing someone's life.

I feel privileged to be able to be the one that first greets the patient at this time, offering support in taking the first huge step to change their whole outlook on dentistry; making them realise that there is a light at the end of the tunnel and not to feel guilty about having this form of treatment carried out. I have read so many articles lately advertising that dental implants can achieve a Hollywood smile - a cosmetic reason to have implants rather that a life changing functional reason. It must be very hard for patients to determine if this is a selfish thing to have done or not.

\section{'The worry of not biting into}

apples in case your

crown or bridgework

becomes loose becomes

a thing of the past.'

\section{A huge difference}

Implants can make a huge difference to people's lives; their quality of life improves as their confidence grows. Going out for a meal is no longer a problem. The worry of their denture moving while eating, avoiding crusty bread 


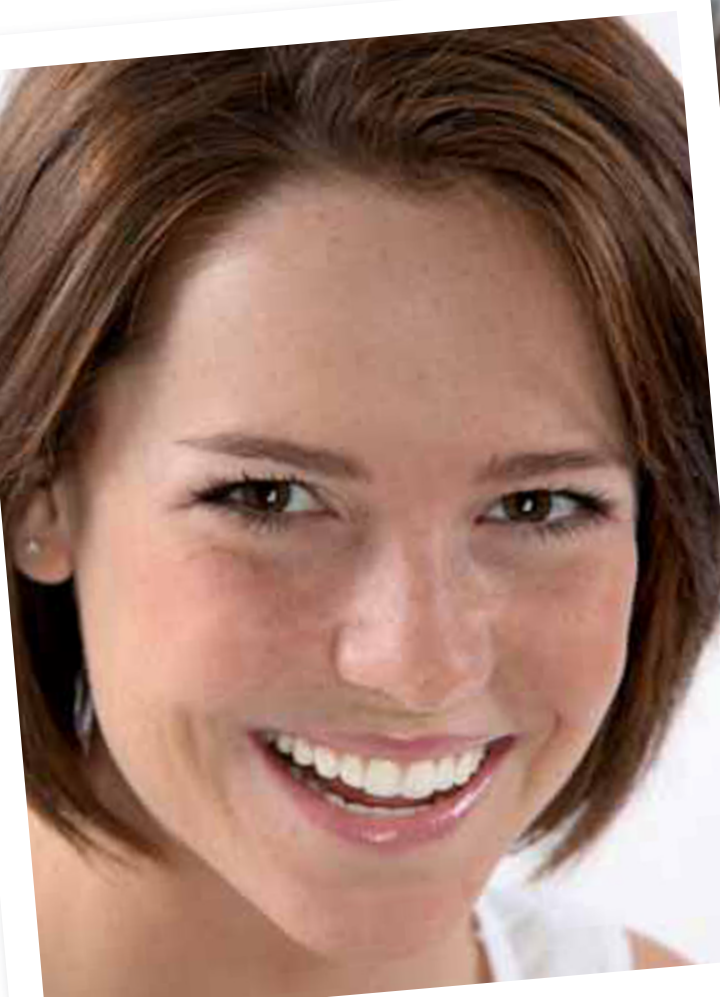

and steaks are a thing of the past. The worry of not biting into apples in case your crown or bridgework becomes loose becomes a thing of the past. All of this improves the patient's quality of life.

\section{The nurse's role}

Nursing for an implantologist is a very rewarding form of dental nursing, it requires a lot of organisation and the ability to stay in theatre for many hours on your feet in one position while retracting, aspirating and reassuring the patient. The dental nurse's role in implantology involves a lot of patient counselling beforehand and throughout the treatment as dental implants can be a long journey for the patient to undertake. Some of our patients may need some ground work carried out before implants can be placed; this requires a lot of skill and patience from the implant team to ensure that the patient fully understands what will be involved. The treatment may involve having a sinus bone graft or a block bone graft if the bone height or width is not adequate enough to place an implant, which can be a further negative for the patient as there will be an extra six months' wait until the implant treatment can begin.

\section{A rollercoaster ride}

There may be no quick fix; you need to be totally honest with the patient before they take this journey. They need to be psychologically prepared to have the work carried out as there will be a lot of negatives for the patient before the positives start.
'I feel privileged to be the one that

first greets the patient, making them

\section{realise that there}

\section{is a light at the end of}

\section{the tunnel.'}

As a dental nurse you have to go through the rollercoaster ride with the patient and bring them out the other side with a smile on their face. Every patient who leaves the practice says the same: 'you have given me my smile back'. It just shows that with implants, although they have to be aesthetically pleasing, the function is the most important thing to a patient who only wants to eat, talk and be confident about their teeth. The only downside to dental implants from most of our patients' points of view is that they gain weight through trying all the food they have missed out on over the years - but when asked they still would not go back to dentures!

At the Dental Implant Clinic we run three week-long Live Skills courses for dentists to learn how to place implants. We also run courses for dental nurses to assist in the placement of a dental implant and how to run an implant practice. These courses have been running for the past four years and have been very successful, receiving positive feedback from the delegates and the patients involved. All of the delegates keep in contact with the practice and it feels good to know I am involved in the teaching of people who wish to start a rewarding career in dental implants.

Dental nurses can find out more about the dental implant nursing course at Nicola's practice by telephoning the Dental Implant Clinic in Bath on 01225448400 and asking for Nicola. Also see the book review on page 17.

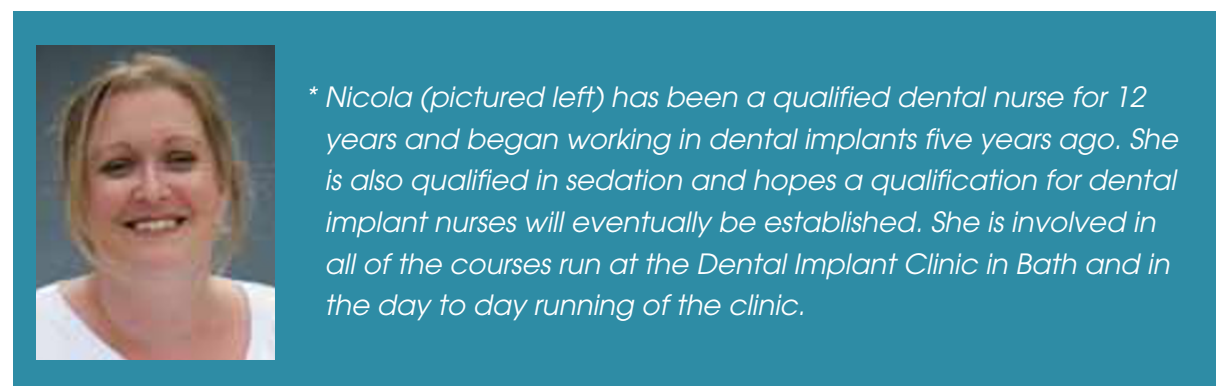

